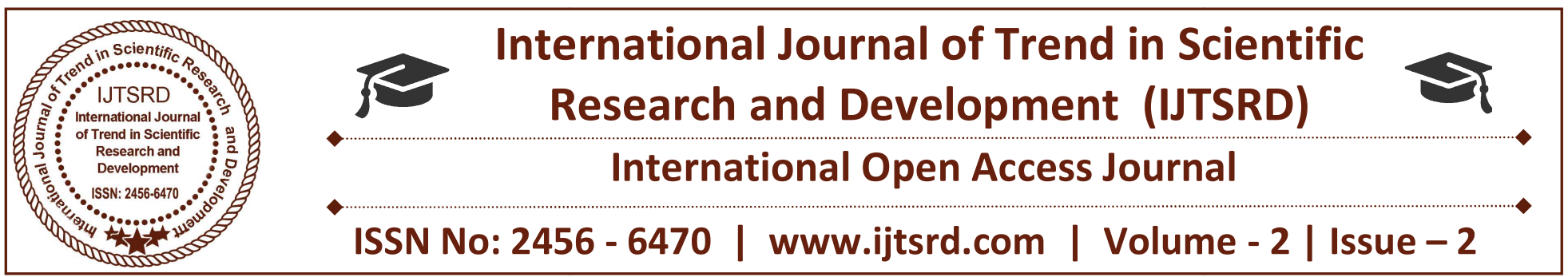

\title{
Review on Detection and Grading the Cataract based on Image Processing
}

\author{
Mrs. R. S. Patil \\ M. Tech. Student, Department of Technology, \\ Shivaji University, Kolhapur, \\ Maharashtra, India
}

\author{
Dr. Uttam L. Bombale \\ Associate Professor, Department of Technology, \\ Shivaji University, Kolhapur, \\ Maharashtra, India
}

\begin{abstract}
This paper gives overview of development of system to detect \& grade the cataract based on image processing.Cataract is when lens gets cloudy. It is leading cause of blindness worldwide. It causes a blurred and foggy vision. Catarct can lead to partial or complete loss of eyesight. A protein layer is developed gradually and the lens becomes cloudy over a long period of time which reduces vision and leads to blindness. Early treatment can lessen the difficulties faced by cataract. Early stage of cataract is not detected by manual method then it will cause problem. Accurate diagnosis and timely treatment of nuclear cataract is essential to prevent vision loss. Severity of cataract is given by grading system. Cataract is classified into three types based on the area of protein deposition named as nuclear cataract, cortical cataract, posterior sub capsular cataract. With the development of image processing techniques, efforts have been put in the development of automatic systems to improve grading objectivity.
\end{abstract}

Keywords: Slit lamp, pupil, grading system, LOCSIII

\section{INTRODUCTION}

CATARACT is a loss of the normal transparency of human lens due to opacity. The lens is formed by of water and protein. A cataract is formed when the protein clumps together. It clouds the lens which reduces the light and produces blurry vision. The leading cause of visual loss is Age-related cataract. The age-related cataract can be classified into three types according to the location of opacity in the lens as nuclear cataract, cortical cataract and post subcapsular cataract. Nuclear cataract as the name suggests develops on the nucleus of the lens. It is normally caused in aged people. Cortical cataract develops in the cortex of the lens which surrounds the lens. At the back of lens Posterior sub-capsular cataract develops. Nuclear cataract is the most common of all cataract types. Clinically, ophthalmologists evaluated the degree of cataract by comparing the picture observed through camera with a series of standard photographs, which is termed as clinical grading such as Lens Opacities Classification System III (LOCSIII).

\section{LITERATURE SURVEY}

Mahmoud Mahlouji and Ali Noruzi [2012][1]represented a human iris recognition system in unconstrained environments in which an effective method is proposed for localization of iris inner and outer boundaries. For iris recognition following steps are used:

1.1. Image acquisition: in this stage, a photo is taken from iris.

1.2. Pre-processing: involving edge detection, contrast adjustment and multiplier.

1.3. Segmentation: including localization of iris inner and outer boundaries and localization of boundary between iris and eyelids. 
1.4. Normalization: involving transformation from polar to Cartesian coordinates and normalization of iris image.

1.5. Feature extraction: including noise removal from iris image and generating iris code.

1.6. Classification and matching: involving comparing and matching of iris code with the codes already saved in database. For Iris inner boundary localization intensity is very different in pupil ary inner and outer parts, and pupil is darker compared with iris, the use of Canny edge detection in pre-processing stage results in determining points in iris pupil boundary.After determining edge points, by the use of circular Hough Transform, the centre and radius of iris circle are obtained. For iris outer boundary localization edge detection algorithms used. Which are able to detect outer iris edges identify those points as edge.It gives effective method to recognize iris boundaries by performing Canny edge detection and Hough transform.

Naveen Singh1, Dilip Gandhi2, Krishna Pal Singh[2011] [2]represented iris recognition system using a canny edge detection and a circular hough transform .The eye detection step of proposed method firstly detects possible eye centre by the circular Hough transform. Then it extracts histogram of gradient from rectangular window centred at each eye centre. Here image acquisition is done by CCD camera. the resolution is set to $640 \times 480$, the type of the image to jpeg, and the mode to white and black for greater details. The image transferred RGB to gray level and from eight-bit to double precision. Before performing iris pattern matching, the boundaries of the iris should be located. We then use the Canny operator with the default threshold value given by Matlab, to obtain the gradient image. We apply a Circular summation which consists of summing the intensities over all circles, by using three nested loops to pass over all possible radii and centre coordinates. The circle with the biggest radius and highest summation corresponds to the outer boundary. After detecting the outer boundary, we test the intensity of pixels within the iris. Each image applied to the Haar wavelet as the combination of six matrices: $\mathrm{cD} 4 \mathrm{~h}$ and $\mathrm{cD} 5 \mathrm{~h}, \mathrm{cD} 4 \mathrm{v}$ and $\mathrm{cD} 5 \mathrm{v}, \mathrm{cD} 4 d a n d \mathrm{cD} 5$. All these matrices are combined to build one single vector characterizing the iris patterns. This vector is called the feature vector. It is very important to represent the obtained vector in a binary code because it is easier to find the difference between two binary code-words than between two number vectors. Test of statistical independence enables the comparison of two iris patterns.

Yasutaka Ito, Wataru Ohyama, Tetsushi Wakabayashi, Fumitaka Kimura [2012][3] represented Detection of Eyes by Circular Hough Transform and Histogram of Gradient. The eye detection step of proposed method firstly detects possible eye centre by the circular Hough transform. Then it extracts histogram of gradient from rectangular window centred at each eye centre. The first is two dimensional Hough transformation for detecting circle of unknown radius. The circular Hough transform first generates two dimensional parameter space (xc; yc) using the gradient of gray scale. The circle with unknown radius can be detected more efficiently. The evaluation of likelihood of eye using histogram of gradient and Support Vector Machine (SVM). Gradient $g$ of gray scale $f$ is given by

$g=(f x ; f y)$

where $\mathrm{fx}$; fy is partial derivative of $\mathrm{f}$ regarding $\mathrm{x}$ and $\mathrm{y}$ respectively.

Circular Hough transform first detects only the centre of the circle with unknown radius on two-dimensional parameter space $(x c ; y c)$. gradient is calculated from the facial image by Sobel filter, and draws a line segment to gradient direction from each edge point in the images. Drawing the line segment to gradient direction generates projection of Hough space (xc; yc; r) to its marginal space (xc; yc). The circle with higher contrast such as iris in white part of the eye contributes more in locating the centre by voting the gradient strength of starting point. The circular transform is smoothed by moving average filter of size $3 \_3$. The possible eyes are detected at each local maximum that is greater than a predefined threshold. The circular transform is smoothed by moving average filter of size 3 - 3 . The possible eyes are detected at each local maximum that is greater than a predefined threshold. To evaluate the effectiveness of the proposed method, evaluation experiments are performed using the image of the FERET database. The images used in the experiment are a gray scale image containing a face in frontal view with no occlusion. The image size is 512 _ 768 pixels, and the faces have high degree of variability in scale, location, slant and expressions and appearances such as 
smiling, closing eyes and wearing glasses. The normalized feature vector is used to evaluate the likelihood of eye by SVM. possible eye detection by circular Hough transformation and likelihood evaluation by SVM using histogram of gradient is more accurate and effective than the other methods.

Amol B. Jagadale and D. V. Jadhav [2016][4]represented Early Detection and Categorization of Cataract using Slit-Lamp Images by Hough Circular Transform.

The shape of pupil is circular, Hough transformed can be used to detect the same and eliminate the iris. This represents two methods. First development of pupil detection algorithm using hough circle detection transform The Hough transform is operated on resized image with Matlab function imfindcircles This algorithm returns possible centres coordinates for detected circles and their radius. If the circles are more than one, by adjusting Input parameters in Hough transform single circle can be obtained. This circle covers maximum pupil area and its centre is at pupil centre. By using viscircle function, the over lapping circle on pupil region \& development of pupil detection algorithm is using region properties. The part pupil region is cropped and maximum and minimum intensity values are found. The region colour based (Intensity Values) separation command from Matlab is used to separate iris and pupil.

Shashwat Pathak and Basant Kumar [2016][5] represented a Robust Automated Cataract Detection Algorithm Using Diagnostic Opinion Based Parameter Thresholding for Telemedicine Application This application uses image processing technique. Firstly patient should visit a primary eye screening centre or they can access the system over a mobile computing device. The cataract screening facility is available by registering on a mobile app. By the technician or a patient the image will be uploaded. These images with patient information will be sent to the server. System will generate report. Then it will be sent to the patient and for secondary opinion to an eye expert. Eye expert will give final decision. By reading texture information from circular pupil it detects presence of cataract. It is not applicable in case of child subjects, also child subjects suffering from coloboma, child subjects who have non circular pupils.

\section{CONCLUSION}

For cataract detection pupil detection is necessary. For the detection of pupil Circular Hough Transform is effective method.

A Robust Automated Cataract Detection Algorithm Using Diagnostic Opinion Based Parameter Thresholding for Telemedicine Application only adults can check the cataract. This limitation can be avoided in proposed system

\section{REFRENCES:}

1. Mahmoud Mahlouji and Ali Noruzi "Human Iris Segmentation for Iris Recognition in Unconstrained Environment", IJCSI International Journal of Computer Science Issues, Vol. 9, Issue 1, No. 3, January 2012.

2. Naveen Singh, Dilip Gandhi, Krishna Pal Singh "Iris recognition system using a canny edge detection and a circular hough transferm", International Journal of Advances in Engineering \& Technology, May 2011.

3. Yasutaka Ito, Wataru Ohyama, Tetsushi Wakabayashi, Fumitaka Kimura "Detection of Eyes by Circular Hough Transform and Histogram of Gradient", 21st International Conference on Pattern Recognition 2012.

4. Amol B. Jagadale and D. V. Jadhav "Early Detection and Categorization of Cataract using

Slit-Lamp Images by Hough Circular Transform", International Conference on Communication and Signal Processing, April 6-8, 2016

5. Shashwat Pathak * and Basant Kumar "Robust Automated Cataract Detection Algorithm Using Diagnostic Opinion Based Parameter Thresholding for Telemedicine Application"Article Academic Editor: Mostafa Bassiouni Received: 29 June 2016; Accepted: 6 September 2016; Published: 15 September 2016

6. Matsoso Samuel Monaheng1, Padmaja Kuruba "Iris recognition using circular hough transform", International Journal of Innovative Research in Science, Engineering and Technology Vol. 2, Issue 8, August 2013.

7. Deepika Prashar, Mnupreet Kaur "Human Eye Iris Recognition Using Discrete 2d Reverse Biorthogonal Wavelet 6.8", International journal 
of scientific \& technology research volume 3,issue 8, August 2014.

8. Huiqi Li, Joo Hwee Lim, Jiang Liu, Paul Mitchell, Ava Grace Tan, Jie Jin Wang, and Tien Yin Wong " A Computer-Aided Diagnosis System of Nuclear Cataract ", IEEE transactions on biomedical engineering, vol. 57, NO. 7, JULY 2010

9. Wei Huang, Kap Luk Chan ,"A Computer Assisted Method for Nuclear Cataract Grading From Slit-Lamp Images Using Ranking", IEEE transaction on medical imaging,vol.30.No.1,January 2011.

10. Huiqi Li, Joo Hwee Lim, Jiang Liu, Damon Wing Kee Wong ,"Feature Analysis in Slit-lamp Image for Nuclear Cataract Diagnosis", 2010 3rd International Conference on Biomedical Engineering and Informatics (BMEI 2010).

11. Rohit Chavan, Arvind Nair, Dheeraj Jadhav, Niranjan Bhat, "Analysis and Study of Cataract Detection Techniques",2016 International Conference on Global Trends in Signal Processing, Information Computing and Communication.

12. Z.He, T.Tan, Z.Sun, and X.Qui, "Towards accurate and fast iris segmentation for iris biometrics", IEEE Trans, On PAMI, Vol. 31, no. 9, pp.1670-1684, Sept. 2009.

13. Niya C P, Jaykumar T.V, "Analysis of Different Automatic Cataract Detection and Classification Methods", IEEE International Advance Computing Conference (IACC), Pages 696-700, DOI: 10.1109/IADCC.2015.754796, 2015.

14. Y. Zhu, T. Tan and Y. Wang, "Biometric personal identification based on iris patterns", Proceedings of the 15th International Conference on Pattern Recognition, Spain, Vol. 2, 2000.

15. N. Ritter, "Location of the pupil-iris border in slitlamp images of the cornea", Proceedings of the International Conference on Image Analysis and Processing, 1999. 